Document downloaded from:

http://hdl.handle.net/10251/120629

This paper must be cited as:

Gomez-Barquero, D.; Navratil, D.; Appleby, S.; Stagg, M. (2018). Point-to-Multipoint Communication Enablers for the Fifth Generation of Wireless Systems. IEEE Communications Standards Magazine. 2(1):53-59. https://doi.org/10.1109/MCOMSTD.2018.1700069

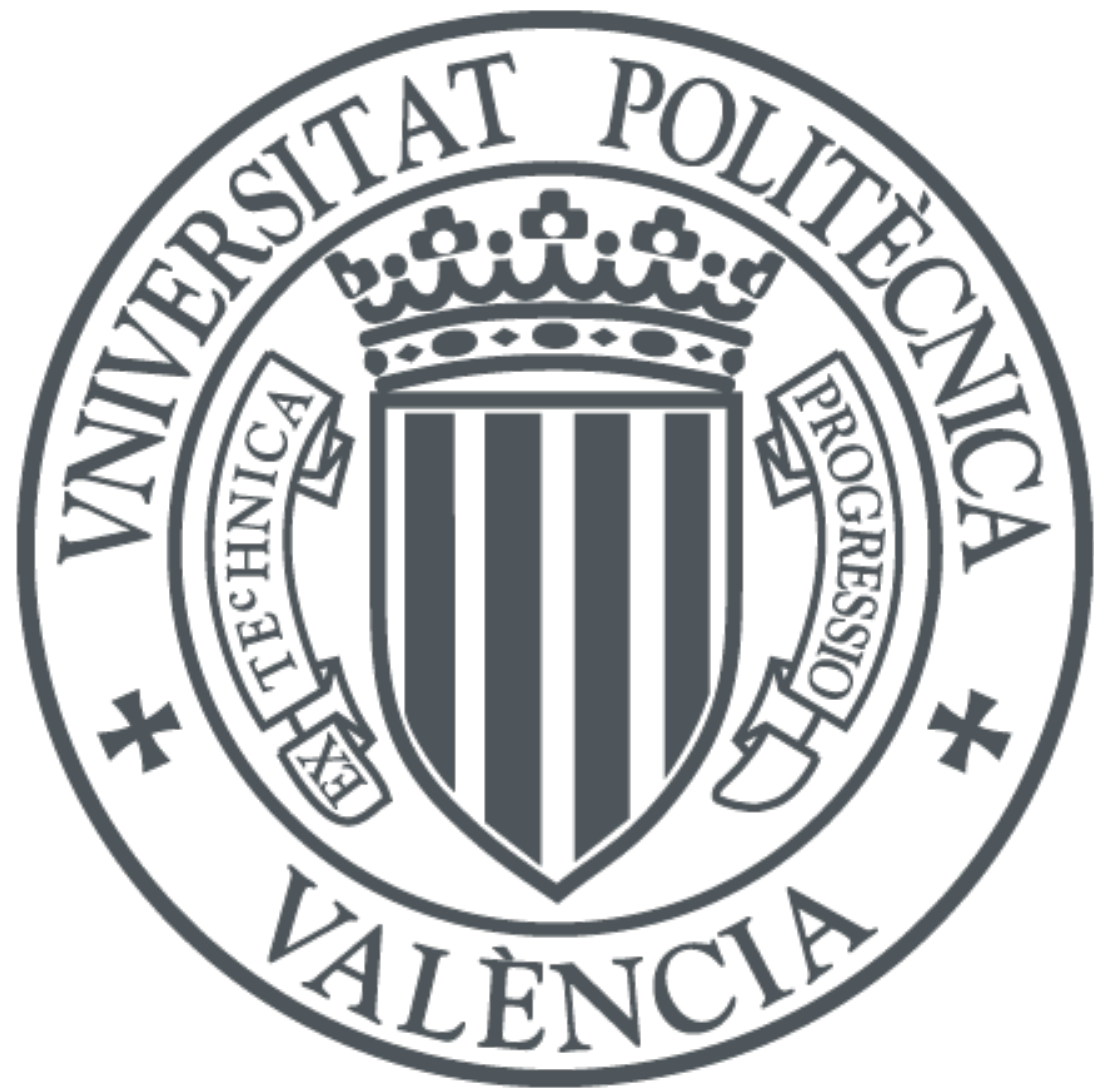

The final publication is available at

http://doi.org/10.1109/MCOMSTD.2018.1700069

Copyright Institute of Electrical and Electronics Engineers

Additional Information

(c) 2018 IEEE. Personal use of this material is permitted. Permission from IEEE must be obtained for all other users, including reprinting/ republishing this material for advertising or promotional purposes, creating new collective works for resale or redistribution to servers or lists, or reuse of any copyrighted components of this work in other works. 


\title{
Point-to-Multipoint Communication Enablers for the Fifth-Generation of Wireless Systems
}

\begin{abstract}
3GPP has enhanced the point-to-multipoint (PTM) communication capabilities of 4G LTE in all releases since the adoption of eMBMS (enhanced Multimedia Broadcast Multicast Service) in Release 9. Recent enhancements cover not only television services, but also critical, machinetype and vehicular communications, following the backwards-compatibility design philosophy of LTE. This paper discusses the opportunity in the design and standardization of $5 \mathrm{G}$ to break with the existing paradigm for PTM transmissions in 4G LTE, where broadcast PTM transmissions were initially conceived as an add-on and pre-positioned service. 5G brings the opportunity to incorporate PTM capabilities as built-in delivery features from the outset, integrating point-topoint (PTP) and PTM modes under one common framework and enabling a dynamic use of PTM to maximize network and spectrum efficiency. This approach will open a door to completely new levels of network management and delivery cost-efficiency. The paper also discusses the implications of PTM for network slicing, to customize and optimize network resources on a common 5G infrastructure to accommodate different use cases and services taking into account the user density.
\end{abstract}

\section{KEYWORKS}

5G, 5G-PPP, broadcast, caching, core network, critical communications, machine-type communications, media delivery, multicast, network slicing, point-to-multipoint, radio access network, vehicular communications. 


\section{INTRODUCTION}

Broadcast is a suitable transport technology to deliver the same content to an unlimited number of people or devices within the covered area with a defined and stable quality of service, without substantially increasing network capacity requirements, energy consumption and costs. In scenarios where a very large number of users or devices consume the same data, such as a popular media content, emergency messages and software updates, the use of multicast/broadcast pointto-multipoint (PTM) schemes instead of unicast point-to-point (PTP) can offer huge capacity gains and sustainable operation of services, ensuring a cost effective high quality delivery mechanism. This also allows valuable network resources to be reused for PTP-only applications.

Broadcast was considered by many stakeholders as an integral part of the 5G system from the beginning [1]. PTM transmissions are key technical components for the new $5 \mathrm{G}$ radio access and core networks, not only for television (TV) services, that have traditionally led the definition of PTM components in 3G and 4G LTE, but also for other vertical industries which role in the recent standardization efforts of eMBMS was equally important. 3GPP has enhanced the PTM capabilities of 4G LTE in all releases since the adoption of eMBMS (enhanced Multimedia Broadcast Multicast Service) in Release (Rel') 9 [2]. Recent enhancements cover not only TV services, but also critical, machine-type and vehicular communications, always keeping the backwards-compatibility design philosophy of LTE.

However, despite the unprecedented research and standardization efforts invested worldwide in 5G, PTM has gathered very little attention so far. 3GPP decided to prioritize the work in other areas for the first release of 5G, Rel'15, and hence 5G PTM would only be addressed starting from Rel'16. The lack of PTM characteristics in the development process of 5G may imply a future limitation of the PTP network infrastructures by not providing a cost-effective and scalable delivery solution, leading to an inefficient service provisioning and utilization of the network and spectrum resources when distributing the same data to multiple users and devices (e.g., live and linear content with very large audiences or mass software updates).

This paper reviews use cases for PTM transmissions in 5G and the evolution of PTM transmissions in 4G LTE, and discusses the opportunity in the design and standardization of 5G to incorporate PTM capabilities as built-in delivery features taking into account the requirements and use cases of different verticals from the outset, integrating PTP and PTM modes under one common framework. This approach will open a door to completely new levels of network management and cost-efficiency delivery. The paper also discusses the implications of PTM for network slicing, that enables to customize and optimize network slices on a common 5G infrastructure to accommodate different use cases and services [3].

The rest of the paper is structured as follows. Section 2 gives a brief overview of PTM use cases in 5G. Section 3 describes the evolution of PTM transmissions in 4G LTE. Section 4 is devoted to elaborate the paradigm change to implement PTM and caching in 5G in a flexible and dynamic way as built-in delivery optimization tools. Section 5 reviews the 5G standardization process and the potential timeline for PTM standardization. Section 6 discusses the implications of PTM for network slicing. The paper is concluded with an outlook in Section 7.

\section{Use CASes for Point-to-Multipoint in 5G}

PTM transmissions can provide a more efficient delivery compared to PTP whenever a service or an application require the same data to be delivered to multiple UEs (User Equipment) at the same time. This is why PTM is considered to be an essential feature for 5G applications in a number of vertical sectors, such as Automotive, IoT, Media \& Entertainment (M\&E) and Public Warning (PW), see Fig. 1 [4]. 


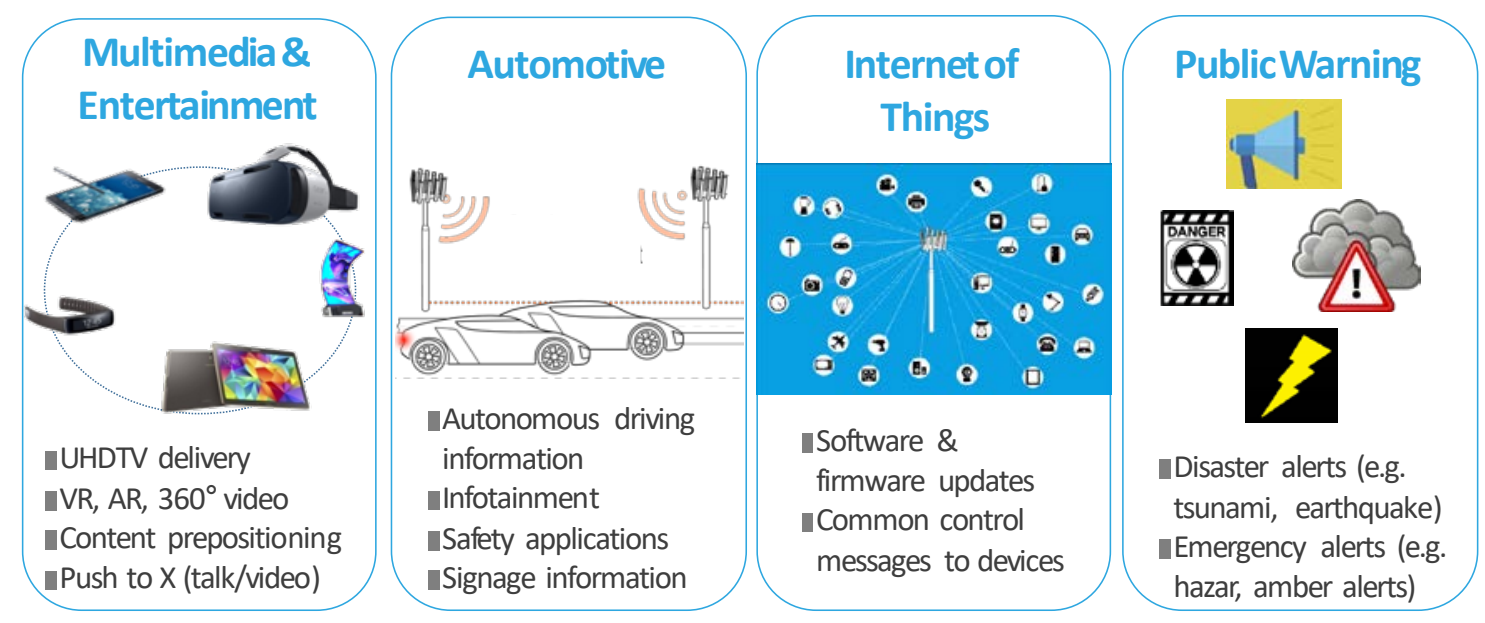

Figure 1: Point-to-Multipoint Use Case Families in 5G. Another use case family is airborne communications (drones and satellite).

In 5G M\&E use cases, PTM will facilitate the distribution of audio-visual media content and services, in particular when covering popular live events that attract large concurrent audiences. PTM network coverage could either be localized to a specific venue or extended to a wide area, potentially the size of a whole country. A novel use case based on 5G PTM could be a dedicated wide-area coverage broadcast network, potentially shared among several network operators, since initial standardization work on this topic has been undertaken in 3GPP Rel'14 for eMBMS [5]. It should be noted that the efficiency gains that might be achieved with PTM will be even more significant when the emerging high quality immersive content formats such as ultra-highdefinition television (UHDTV), 360-degree video, virtual and augmented reality (VR/AR) become more prevalent.

5G PTM in combination with caching capabilities would facilitate time-shifting and non-realtime media consumption. At the same time, pre-emptive delivery of content to the storage in the device can help to balance the network load over time and reduce expensive capacity overprovisioning, hence making the delivery more cost-efficient. This method could be applicable to non-time-critical information services (e.g. news alerts) that could be conveniently 'pushed' (content pre-positioning) to the devices rather than being 'pulled' by the end users at the time of the consumption. Another M\&E use case for 5G PTM IP-based TV production, with very stringent requirements in particular on throughput, latency, time synchronisation, and reliability.

For IoT use cases, 5G PTM could facilitate the delivery of common data such as software and operating system updates to a large number of connected devices. In these cases, the main 5G requirements are very high reliability and, for some devices, low power consumption. Scheduled PTM updates would allow operators to perform non-time-critical broadcasting during off-peak times without negatively affecting PTP network traffic. These use cases are also relevant for connected 5G smart cities and a variety of industrial applications.

There are a number of automotive use cases where 5G PTM transmissions can play a role, such as delivering media content, entertainment and information services, navigation data, road safety information and traffic services. Other examples include software updates in the vehicles. The main 5G technical requirements for vehicular communications are related to throughput, latency, reliability, and velocity.

A key requirement for PW applications is a secure and reliable delivery of alert messages to general population in emergency situations. 5G PTM capabilities would ensure that PW messages reach a large number of users simultaneously without causing network congestion or even significantly increasing the traffic load. 
5G PTM use cases also cover airborne communications, including drones and satellites. The use of satellites is key to support ubiquitous coverage (for disaster relief, broadcast services and remote sensor connectivity), and it is expected that 5G will support highly available and reliable connectivity using satellites. Applications envisaged for drones include live broadcast of outdoor events, where the high quality video is transmitted from the flying drone to the TV station via the 5G mobile network. In this case, the technical requirements consist of providing continuous wireless coverage for a flying UE at low altitude with a moderate speed.

\section{Point-to-Multipoint Evolution in 4G LTE}

4G LTE was originally designed to support broadcast services. The technology eMBMS, also known as LTE Broadcast, was introduced in Rel'9 (recall that the initial LTE standard was introduced in 3GPP Rel'8). eMBMS introduced new PTM radio bearers and multicast support in the core network with small changes to the existing radio and core network protocols of LTE [2]. New physical, transport and logic channels were defined as well as three new logical entities in the core network architecture. The BM-SC (Broadcast Multicast Service Center) is the entry point for content and is responsible for, among other things, service announcement, configuration of transmission and user authentication and encryption. The eMBMS gateway distributes the MBMS data to base stations using IP multicast and generates session control signaling for the mobility management entity. The MCE (Multi-cell/Multicast Coordination Entity) allocates radio resources in time and frequency for eMBMS and decides the configuration of the radio transmission.

eMBMS Rel'9 was largely based on the original MBMS technology standardized for 3G in Rel'6 [6], which was initially conceived as an add-on mobile TV service in a large pre-planned area with a rather static configuration. For example, the initial eMBMS specification does not allow to dynamically adjusting the service area, also known as MBSFN (MBMS over Single Frequency Networks) area, according to the UE distribution, since it is e.g. configured by operation and maintenance [2]. Another example is the static configuration of the eMBMS resource allocation because the bearer setup and modification time was rather long, in the order of few seconds. eMBMS data transmission is time multiplexed with other LTE unicast traffic, and up to 6 of the 10 subframes of a radio frame are configurable for eMBMS. eMBMS transmissions also occupy the entire system bandwidth, and multiplexing with unicast in the same subframe is not allowed even though not all the radio resources in frequency domain are utilized.

Another impending problem for the deployment of LTE Broadcast is that not all commercial devices support eMBMS, and the ones that do require an upgrade to the firmware to activate it. Also, new equipment is needed in the core, which requires investment from the operator side.

Since its introduction in Rel'9, eMBMS has continued to evolve in all subsequent releases. eMBMS has gone through a very significant set of enhancements, with major changes to architecture concepts and major enhancements to the Radio Access Network (RAN). Rel'14 is very different from the first version of eMBMS developed in Rel'9, but it carries a long legacy due to the backwards-compatible design philosophy of $4 \mathrm{G}$ LTE. The improvements can be generally classified around two areas: enhancements for the delivery of television services to deploy stand-alone eMBMS broadcast networks, and generic delivery efficiency enhancements using PTM as an internal and dynamic delivery optimization tool. Fig. 2 depicts the evolution of PTM transmissions in 4G LTE. The main enhancements are briefly explained next. 


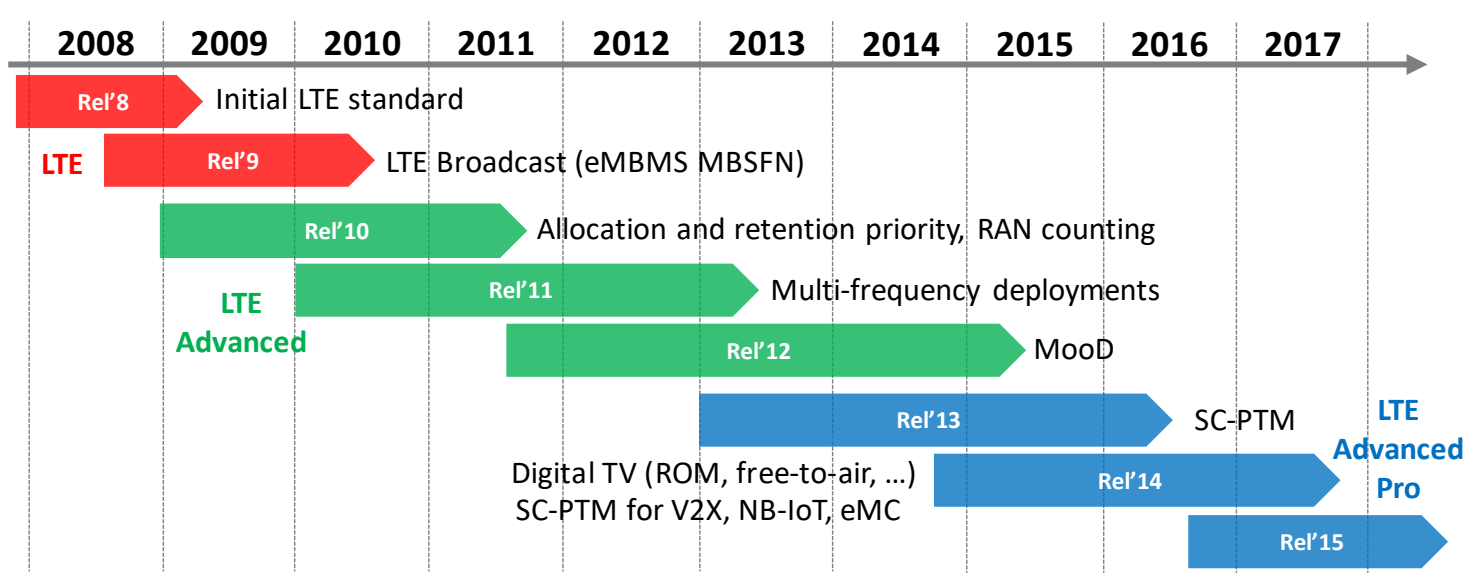

Figure 2: Point-to-Multipoint Evolution in 4G LTE. LTE-Advanced (Rel'10, '11 and '12) and LTE-Advanced Pro (Rel'13 and '14).

Rel'10 introduced a RAN-based counting of UEs in connected mode interested in an MBMS service. This release also allowed the use of unused MBSFN subframes for unicast reception, and enhanced the admission control for MBMS sessions by the introduction of the allocation and retention priority session parameters.

Rel'11 introduced service acquisition and continuity in multi-frequency deployments where the MBMS service is provided via more than one frequency [7].

Rel'12 introduced one of the main enhancements of eMBMS: MooD (MBMS operation on Demand), which enables automatic and seamless MBMS service activation and deactivation based on the UEs' service consumption reporting [8], [9]. Rel'12 also introduced improvements in the physical measurements (e.g., signal power, error rates) the UEs can be ordered to perform for MBSFN network optimization, and specified eMBMS support for critical communications (in particular, as part of the group communication service enablers).

Rel'13 introduced another of the main enhancements to eMBMS to increase the resource allocation flexibility for PTM: SC-PTM (Single-Cell PTM) [10]. SC-PTM allows one cell to broadcast the same content to a group of UEs multiplexing broadcast and unicast data on the same physical downlink shared channel (PDSCH) instead of using for broadcast a different and dedicated physical channel, the physical multicast channel ((P)MCH). This allows a very flexible and dynamic radio resource allocation for broadcast transmissions, equivalent to unicast. Furthermore, it also benefits of a reduced end-to-end latency. SC-PTM could also exploit the unicast feedback for advanced link adaptation schemes such as adaptive modulation and coding for groups with a small number of UEs [11]. However, this feature was finally not standardized in Rel'13. SC-PTM reuses eMBMS architecture and core network procedures and partially reuses eMBMS procedures in RAN.

Rel'14 introduced MBSFN and SC-PTM for V2X (vehicular to everything) communications and SC-PTM for IoT solutions eMTC (enhanced Machine-Type Communication) and NB-IoT (NarrowBand-IoT). Rel'14 also introduced many features to enhance the delivery of television (TV) services with eMBMS, to expand the reach of MBMS into traditional TV receivers and to enable the deployment of dedicated broadcast eMBMS networks supporting public broadcasting requirements. Services provided may be distributed in such a way that they can be received by all, including those who are not mobile subscribers. This extends the applicability of mobile broadcast to support public broadcasting requirements.

Architecture enhancements of eMBMS in Rel'14 include new device modes, such as the receive only mode (ROM) for devices without SIM card or 3GPP subscription; new service types to 
enable free-to-air content broadcast that can be received by all, including devices without subscription, as well as interactive services with ROM devices; an open standardized broadcasting application programming (xMB) external interface towards the TV content providers; transportonly (pass-through) MBMS bearer service type to use the eMBMS network as content delivery platform in the native format without transcoding; and shared networks among several MNOs to avoid broadcasting the same content at the same time over different networks and improve the radio resource utilization. The combination of receive only mode devices and shared MBMS architecture enables the deployment of standalone eMBMS broadcast networks. Fig. 3 shows a simplified eMBMS architecture for TV services with a new eMBMS Application Programming Interface (MBMS-API) that has been introduced to simplify access to complex eMBMS procedures.

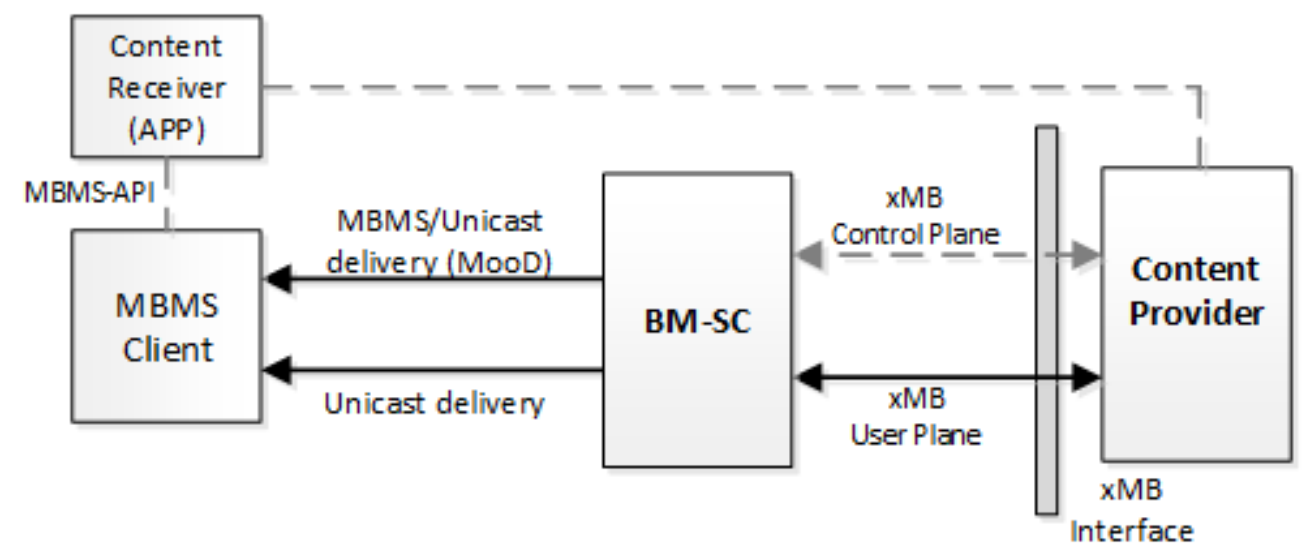

Figure 3: Simplified architecture for TV services over 3GPP eMBMS Rel'14.

Radio access enhancements of eMBMS in Rel'14 include dedicated carriers with up to $100 \%$ MBMS allocation and self-contained system information and synchronization signals (including a new type of MBSFN subframe without unicast control region to reduce the signalling overhead), and a $200 \mu$ s long cyclic prefix to support large inter-site distances. It should be noted that these improvements are not backwards compatible with previous releases, meaning that pre-Rel'14 UEs cannot receive any service from cells operating with these improvements, and hence they are only suitable for new Rel'14 deployments.

\section{The Change of Paradigm for PTM Transmissions in 5G}

PTM transmissions are considered a key technology enabler for 5G, and it is generally acknowledged that an enhanced form of MBMS is required for 5G. The 3GPP SMARTER (New Services and Markets Technology Enablers) study item [12] identified several PTM use cases that cannot be met by 4G LTE including, connected vehicles, in-network and device caching, broadcast services using a dedicated radio carrier and drones. 5G has the opportunity to break with the existing paradigm for PTM transmissions in 4G LTE, where broadcast PTM transmissions were initially conceived as an add-on and pre-positioned service (note that the last "S" in eMBMS stands for Service).

PTM should be implemented in 5G in a flexible and dynamic way as an essential delivery tool needed for optimal system performance. PTM transmissions should be built-in features integrated into the overall system architecture, keeping as many commonalities as possible with unicast PTP in order to maximize compatibility and interoperability, and enable dynamic seamless switching between different modes of operations, as well as their parallel usage. This approach will also enable the deployment of stand-alone 5G broadcast networks for the distribution of radio and TV broadcast services. Furthermore, by adopting a unified approach for PTP and PTM media delivery, it will also simplify the use of broadcast and make it transparent in a straightforward and 
scalable manner to content providers in both dynamic and pre-positioned media delivery scenarios.

The integration of PTP and PTM modes under one common framework is essential to exploit network and spectrum resources in an efficient and economic manner, e.g. a common framework ensures that the use of PTP and PTM modes can be managed transparently within the network, which leads to simplified and unified service design across various access networks. But a common framework also fosters that all network and user equipment support the PTM delivery feature. The fact that specific hardware is required for LTE Broadcast, at both terminal and network sides, is probably the main barrier that has held up investment in the technology.

In this sense, 5G PTM could follow SC-PTM principles in LTE, using the same physical channel as unicast, enabling an agile scheduling of the radio resources for PTM transmissions. But it should remove the inefficiencies of MBSFN and SC-PTM (e.g., ideally, the use of PTM should be dynamically decided on a cell basis, with the possibility of synchronizing groups of adjacent cells to transmit in SFN mode. Also, when broadcasting to a group of few UEs it should be possible to exploit feedback)

\section{5G Standardization Progress ANd 5G PTM}

3GPP started in March 2017 the normative work for 5G in Rel'15, also known as New Radio (NR), focusing on a PTP network infrastructure solution and the early 5G deployments. 3GPP Rel'16 work started in 2018 targeting the ITU (International Telecommunications Union) IMT2020 (International Mobile Telecommunication) submission. Three usage scenarios are defined in the IMT-2020 process: enhanced Mobile Broadband (eMBB), Ultra-Reliable and Low Latency Communications (URLLC), and massive Machine-Type Communications (mMTC). 3GPP plans to submit both LTE-Advanced Pro and 5G-NR as IMT-2020 candidates. 5G-NR primarily targets the eMBB use case, although some URLLC related features will be also considered. The mMTC use case will be covered by the 4G LTE-Advanced Pro based solutions eMTC and NB-IoT that support SC-PTM.

The support of PTM capabilities in 5G will probably happen in Rel'17. In Rel'15 there is an SA4 study item on 5G enhanced Mobile Broadband Media Distribution to study, among other topics, the implications of the $5 \mathrm{G}$ architecture on MBMS services. In 3GPP RAN there were several study item proposals on 5G MBMS for Rel'15, but the very tight schedule of 5G-NR and the high workload of the RAN1 group blocked new study items. A consensus proposal for a Rel'16 study or work item will be presented and evaluated in June 2018. The SA2 group has two new studies items in Rel'16 on IoT and V2X that could cover PTM capabilities. However, developing PTM solutions separately in different studies items could lead to fragmented solutions and higher system complexity. A common PTM framework should be designed to support different applications and verticals, including M\&E.

Backward compatibility was one of the key design requirements for LTE systems, which limited the system design flexibility due to the usage of always-on signalling such as cell-specific reference signals, synchronization signals, etc., which were defined as mandatory requirements in LTE Rel'8. Due to such mandatory requirements, the eMBMS system required the definition of special MBSFN subframes. But, 5G is being designed following a forward compatibility principle in both radio and protocol design. This will ensure smooth introduction of future services and features in subsequent releases of the $5 \mathrm{G}$ specification with no impact on the access of earlier services and UEs. Hence, the opportunity to incorporate PTM transmissions into 5G as built-in delivery optimization feature is still possible.

3GPP should pursue a 5G PTM specification keeping as many commonalities with the current 5G PTP developments. The number of new elements for PTM should be restricted and only added if 
well justified in order to retain the maximum compatibility and ensure full integration and operability. But on-going standardization work should ensure forward compatibility of the overall 5G wireless system to enable PTM communication capabilities to not hamper the potential business models from the start.

\section{NETWORK SLICING AND PTM IN 5G}

Network slicing allows new ways to design, run and optimize networks which should lead to even better utilization of network and computation infrastructure. Network slices can provide exactly the necessary network functions and optimizations to meet the needs of a particular service and possibly even a particular use case within a service. 3GPP defines three slice/service types in Rel'15: eMBB, URLLC and MIoT (massive IoT), see Fig. 4 [13].

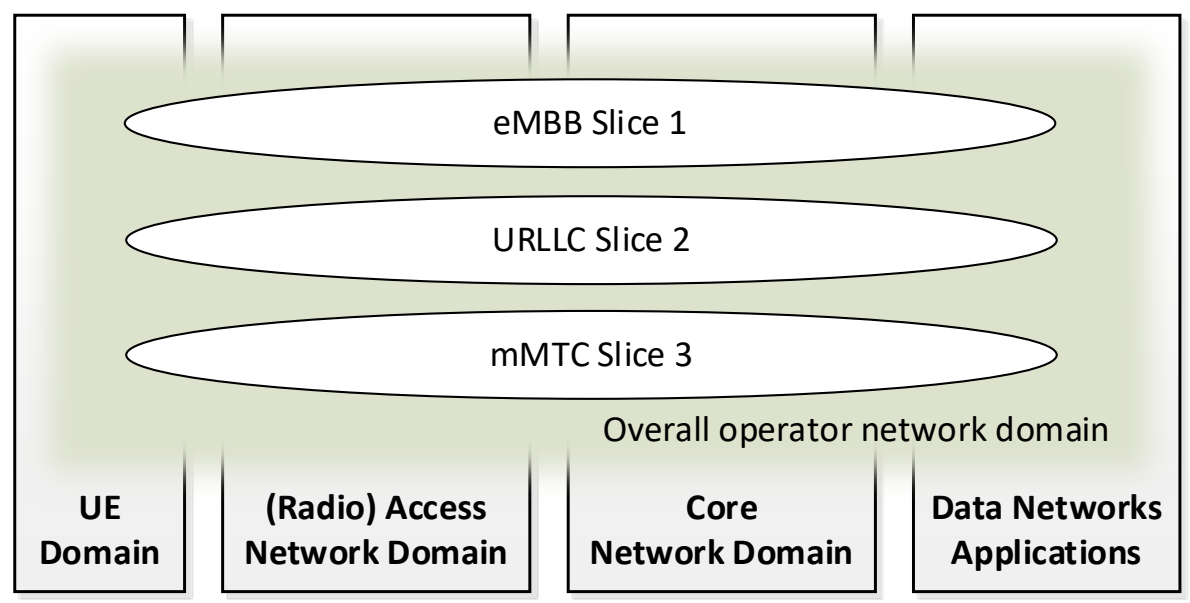

Figure 4: Network slicing concept with three slice types.

Network slicing builds on the foundations laid down by SDN (Software Defined Networking) and NFV (Network Function Virtualization) [3]. SDN revolutionizes the networking by the introduction of a new abstraction layer, the control layer, on top of a common infrastructure layer with well-defined interfaces between the layers: infrastructure, control and application. NFV separates the network functions from hardware and allows the network functions to be run in software in virtualized environment. SDN and NFV both aim at reducing CAPEX and OPEX but most importantly they deliver agility and flexibility in a way that the network management complexity does not scale linearly with the size of the network. The networks become programmable, which enables faster innovation and accelerates time-to-market.

Fig. 5 shows the $5 G$ reference service-based architecture that consists of a number of Network Functions (NF) that has been designed with the key principles, among others, of enabling deployments to use NVF/SDN techniques, and of modularizing the function design in order to enable flexible and efficient network slicing [13]. The 5G service-based architecture will represent a disruptive change compared to existing 4G LTE networks. The main differences lie in the control plane where, instead of predefined interfaces between elements, the procedures (set of interactions) between network functions are defined as NF services, such that control plane network functions communicate with each other using NF services. A NF service consists of operations based on either a request-response or a subscribe-notify models. 


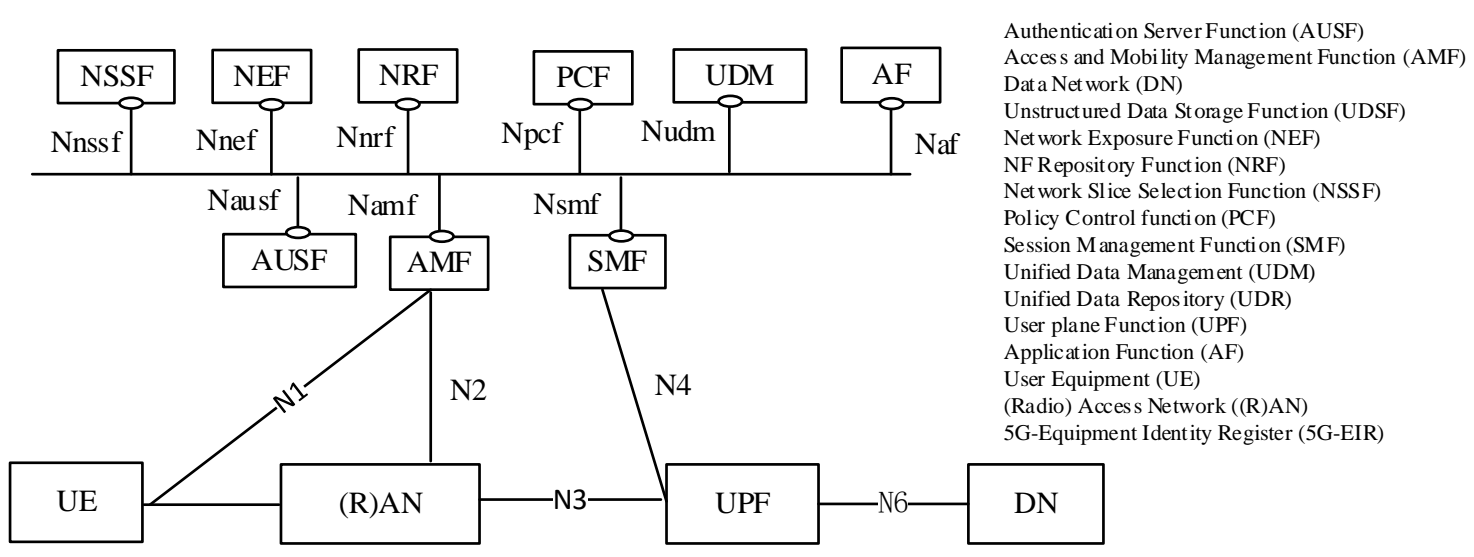

Figure 5: Service-based $5 G$ architecture and defined network functions.

PTM should become a delivery configuration feature of 5G network slicing, such that a dedicated network slice can provide $5 \mathrm{G}$ broadcast services or a network slice (e.g. eMBB) is enhanced with PTM to provide the most optimal content delivery method. But PTM shall not be understood to be a single network function. New functions, including the software functions of the BM-SC, MBMS Gateway and MCE in 4G LTE, will have to be introduced in the end-to-end 5G architecture to support PTM such that 5G network slices are optimized based not only on service performance characteristics as capacity, latency and speed; but also depending on the popularity of the content use PTP and/or PTM delivery. The functional split of the PTM feature should be carefully designed in order to enable the most efficient network slicing. One of the main technical challenges is to design a flexible reconfigurable end-to-end mobile network architecture capable of handling scalable guaranteed latency and reliable end-to-end communications, efficiently integrating caching policies, and leveraging the concept of network slicing for a multi-service $5 \mathrm{G}$ system with PTM capabilities.

The above principles of PTM implementation with 5G network slicing can be applied to the 3GPP 5G network architecture [13]. In the following we consider two different PTM use cases to provide more details about the practical implementation: broadcast in a geographical area and transport optimization for over-the-top media streaming.

The broadcast in geographical area is a general PTM use case which becomes relevant in, for example, network-to-vehicle communication, public warning systems or IoT when the requirement is to dessiminate information to all UEs in a geographical area. The network may deploy a dedicated broadcast network slice to support PTM with its own single network slice selection assistance information (S-NSSAI). The UE may still need to select the network slice but the UE does not receive PTP services. The benefits of this approach are optimization of NFs and PTP and PTM function separation with a common AMF instance for the dedicated broadcast network slice and any other slices the UE may be registered to.

The dedicated broadcast network slice is not suitable approach if the system is to be optimized for varying end-to-end system characteristics such as user's demand for content, as happens with the over-the-top media streaming use case. The change in system characteristics, in this case driven by user's demand, can result in the optimization of the network slice. The network slide selection is not impacted and the UE should not be aware of changes in the network slice instance. For example, when multiple UEs served by a single eMBB network slice consume the same live stream as over-the-top service, the system could enable PTM and switch from PTP to PTM delivery using mechanisms such the 3GPP feature MBMS operation on demand [8] and HTTP over multicast QUIC [14]. To enable PTM may mean to instantiate new network functions in user plane, e.g. a network function for the ingress of multicast content, and control plane. Considering 
that the supported features and network function optimizations of a network slice may change during its life cycle, then the network slice instance may be seen as a composition of sub-network instances where one sub-network instance could provide the feature PTM transport. The concept of sub-network instances is described in [15] and illustrated in Fig. 5. We propose to expand the concept and include at least the posibility to update the network slice instance by updating the network slice blueprint as necessary during the network slice instance life cycle addressing changes in end-to-end system characteristics. The update of network slice blue print may result in the instantiation or termination of sub-network instances and the change of network slice instance composition. The composition of network slice instance could be unkown to the UE such that the changes to the network slice instance are transparent to the UE.

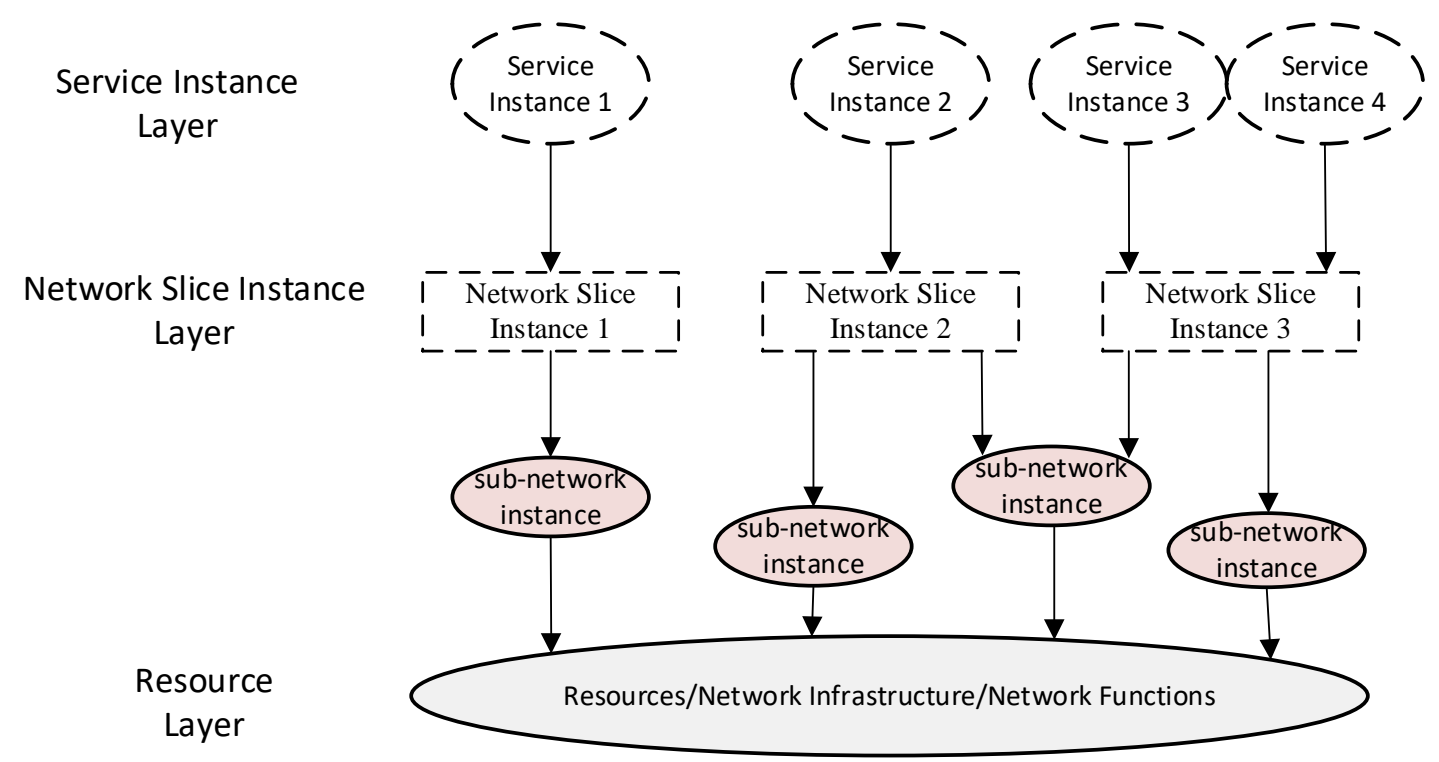

Figure 6: Network slicing concept with sub-network instances.

\section{OUTLOOK}

The introduction of point-to-multipoint capabilities in 5G will increase the efficiency of the delivery of the same content to multiple users or devices. 5G broadcast/multicast should go beyond the current situation whereby LTE Broadcast exists solely as a separate, isolated service. PTM should be implemented in 5G in a flexible and dynamic way as an essential delivery tool of 5G network slicing, such that PTM transmissions become built-in features integrated into the overall 5G system architecture. This approach will not only enable the delivery of 5G broadcast services using dedicated network slices, but it will also enable a dynamical seamless switching between PTP and PTM modes, as well as their parallel usage (e.g., in different areas) for a single service (network slice).

5G should provide a unified PTM framework for relevant verticals and applications, including automotive, IoT, media and entertainment, and public warning. The 5G PTM solution should not only fulfil the technical requirements of the M\&E vertical, such as extremely high data rates for ultra-high fidelity media, but also consider other novel KPIs from the other verticals such as very low latencies, reduced receiver power consumption and ultra-high transmission reliability.

\section{ACKNOWLEDGMENTS}

This work was supported in part by the European Commission under the 5G-PPP project Broadcast and Multicast Communication Enablers for the Fifth-Generation of Wireless Systems 5G-Xcast (H2020-ICT-2016-2 call, grant number 761498). The views expressed in this contribution are those of the authors and do not necessarily represent the project. 


\section{REFERENCES}

[1] NGMN 5G Initiative, “5G White Paper,” White Paper, Feb. 2015.

[2] J. Huschke and M.-A. Phan, "An Overview of the Cellular Broadcasting Technology eMBMS in LTE,” in Next Generation Mobile Broadcasting, pp. 223-252, March 2013.

[3] K. Samdanis, S. Wright, A. Banchs, A. Capone, M. Ulema, K. Obana, "5G Network Slicing - Part 1: Concepts, Principles, and Architectures,” IEEE Communications Magazine, vol. 55., no. 5, pp. 70-71, August 2017.

[4] 5G-PPP 5G-Xcast project, "Definition of Use Cases, Requirements and KPIs," Deliverable D2.1 v1.0, October 2017.

[5] G. K. Walker, J. Wang, C. Lo, X. Zhang, and G. Bao, "Relationship Between LTE Broadcast/eMBMS and Next Generation Broadcast Television," IEEE Transactions on Broadcasting, vol. 60, no. 2, pp. 188-199, June 2014.

[6] F. Hartung, U. Horn, J. Huschke, M. Kampmann, T. Lohmar, and M. Lundevall, "Delivery of Broadcast Services in 3G Networks," IEEE Transactions on Broadcasting, vol. 53, no. 1, pp. 188-199, March 2007.

[7] D. Lecompte and F. Gabin, "Evolved Multimedia Broadcast/Multicast Service (eMBMS) in LTE-Advanced: Overview and Rel-11 Enhancements," IEEE Communications Magazine, vol. 50, no. 11, pp. 68-74, Nov. 2012.

[8] 3GPP TR 26.849 v12.1.0, "Multimedia Broadcast/Multicast Service (MBMS) improvements; MBMS operation on demand,” June 2015.

[9] 3GPP TS 26.346 v14.4.0, "Multimedia Broadcast/Multicast Service (MBMS); Protocols and Codecs”, Sept. 2017

[10] 3GPP TR 36.890 v13.0.0, "Study on Support of Single-Cell Point-to-Multipoint Transmission in LTE,” July 2015.

[11] 3GPP TR 22.891 v14.2.0, "Study on New Services and Markets Technology Enablers," Sept. 2016.

[12] A. Awada, D. Navrátil and M. Säily, "A Study on Single-Cell Point-to-Multipoint Transmission for Public Safety Communications with eMBMS LTE Networks," Proc. IEEE Wireless Communications and Networking Conference (WCNC), Doha, Qatar, 2016.

[13] 3GPP TS 23.501 v15.0.0, “System Architecture for the 5G System; Stage 2,” Dec. 2017.

[14] L. Pardue and R. Bradbury, "Hypertext Transfer Protocol (HTTP) over multicast QUIC," IETF Internet-Draft draft-pardue-quic-http-mcast-01, Aug. 2017.

[15] NGMN 5G Initiative, "Requirements \& Architecture; Work Stream End-to-End Architecture; Description of Network Slicing Concept,” White Paper, Sept. 2016. 


\section{AUTHORS’ BIOGRAPHIES}

DAVID GOMEZ-BARQUERO (dagobar@iteam.upv.es) received a Ph.D. degree in telecommunications engineering from the Universitat Politècnica de València (UPV), Spain. He is a senior researcher (Ramon \& Cajal Fellow) at UPV's Institute of Telecommunications and Multimedia Applications (iTEAM), where he leads a research group working on next-generation wireless broadcast technologies. Dr. Gomez-Barquero has been during the last decade actively participating in the digital television standardization, including DVB-T2, T2-Lite, DVB-NGH, and ATSC 3.0. His recent research interests are focused in the development of broadcast and multicast technology components for 5G. He is the project manager of the 5G-PPP project 5GXcast.

DAVID NAVRÁTIL (david.navratil@nokia-bell-labs.com) received an M.Sc. in electrotechnical engineering and computer science from Brno University of Technology in Czech Republic and an M.Sc. in computer science from University of Joensuu in Finland. He is a senior research specialist at Nokia Bell Labs in Finland. His research focuses on end-to-end mobile network solutions for 4G and 5G systems. He actively contributes to 3GPP standardization. He represented Nokia and later Renesas Mobile Corporation as a delegate in 3GPP between 2005 and 2013 covering TSG GERAN, GERAN2 and SA2.

STEVE APPLEBY (steve.appleby@bt.com) holds a B.Sc. (hons) in Physics from the University of Surrey, and a Ph.D. in Electronics from the University of Kent. Within BT Research, he has led teams on a wide range of topics, including Cybernetics, Computational Linguistics and, most recently, TV and Video technology. Current responsibilities span research on new transport protocols for video streaming, Adaptive Bitrate Streaming over multicast networks, High Dynamic Range video research as well as product-focused technology strategy, including Set Top Box middleware, working with TV vendors on short and longer-term opportunities for IP delivered TV services and technology for content protection.

MATT STAGG (matt.stagg@ee.co.uk) has over 25 years' experience in telecoms, media and entertainment and was recently voted in the top 50 most influential people in online TV for the fourth year running. In 2017, he was awarded the TV Connect People's Choice award for the 'Person who made the biggest impact on the connected entertainment industry'. Within EE he is Head of Media \& Entertainment Technology, developing the strategy for the Broadcast, Media \& Entertainment over 4G / LTE-B \& 5G. This encompasses a 'glass to glass' approach incorporating both contribution and distribution of media. Matt is also the Founder and Chairman of the Mobile Video Alliance. 